\title{
Desmoplastic small-round-cell tumor of the abdomen: A report of two rare cases
}

\author{
JIAJIA CHEN ${ }^{1,2}$, JIFANG SHENG $^{1,2}$, LIJUN WANG $^{3}$, ZHAO-MING WANG $^{3}$ and LANJUAN LI ${ }^{1,2}$ \\ ${ }^{1}$ State Key Laboratory for Diagnosis and Treatment of Infectious Diseases, First Affiliated Hospital, College of Medicine, \\ Zhejiang University; ${ }^{2}$ Collaborative Innovation Center for Diagnosis and Treatment of Infectious Diseases; \\ ${ }^{3}$ Department of Pathology, First Affiliated Hospital, College of Medicine, Zhejiang University, \\ Hangzhou, Zhejiang 310003, P.R. China
}

Received September 28, 2014; Accepted May 15, 2015

DOI: $10.3892 / 01.2015 .3357$

\begin{abstract}
Desmoplastic small-round-cell tumor (DSRCT) is an uncommon type of malignancy, which primarily occurs in adolescent males and develops in the abdominal cavity. The present study reports the case of two manifestations of DSRCT complicated with other diseases, which involved the invasion of the pelvis or abdominal vessels. The first case was of a 25-year-old man with repeated diarrhea and abdominal distension for 9 months; the second case was of a 68-year-old man who presented with persistent abdominal pain for 20 days. In each patient, a mass was identified in the abdomen and biopsies were performed in order to reach a diagnosis. Immunohistochemical staining of the biopsy material revealed a diagnosis of DSRCT in each case. In addition, the present study aimed to discuss the use of imaging techniques in providing evidence for the early diagnosis of DSRCT.
\end{abstract}

\section{Introduction}

Desmoplastic small-round-cell tumor (DSRCT) is an uncommon and aggressive malignancy with a poor prognosis, which is predominantly diagnosed in adolescent males $(1,2)$. The 3- and 5-year survival rates of patients with DSRCT were reported to be $44 \%$ and $15 \%$, respectively (3). It primarily develops in the abdominal cavity. Gerald and Rosai (1) first reported the occurrence of DSRCT in 1989 and characterized it as a distinct type of small-round-blue-cell tumor, which mainly occurs on serosal surfaces, including the peritoneum and tunica vaginalis. In addition, DSRCT was reported to primarily affect Caucasian males in the second or third decade of life $(1,2)$. In general, the manifestations of intra-abdominal

Correspondence to: Professor Lanjuan Li, State Key Laboratory for Diagnosis and Treatment of Infectious Diseases, First Affiliated Hospital, College of Medicine, Zhejiang University, 79 Quingchun Road, Hanzhou, Zhejiang 310003, P.R. China E-mail: 1jli@zju.edu.cn

Key words: education, gastrointestinal cancers, radiology
DSRCT are nonspecific and may present with signs and symptoms that include vague abdominal discomfort or a palpable abdominal mass (4). It can be distinguished from other type of small-round-cell tumors, including neuroblastoma, Ewing's sarcoma and Wilms' tumor, based on its clinical, immunohistological and cytogenetic characteristics.

DSRCT treatments may comprise chemotherapy, surgical resection and radiation therapy, however, survival rates are low. Certain targeted therapies, such as IGF-1R inhibitors, mTOR inhibitors and androgen blockade, have been suggested to provide a possible clinical benefit. In addition, polychemotherapy, surgery combined with hyperthermic intraperitoneal chemotherapy, or radio-immunotherapy may also be promising strategies for long-term disease control of DSRCT (5).

The present study describes the manifestations of two cases of DSRCT that were complicated with other diseases and involved the invasion of the pelvis or abdominal vessels. The two cases were initially misdiagnosed, prior to immunohistological confirmation. Written informed consent for the publication of these case reports was obtained from the patient or the patient's family.

\section{Case reports}

Case 1. A 25-year old man was admitted to the First Affiliated Hospital, College of Medicine, Zhejiang University (Hangzhou, China) in May 2008 after presenting with repeated diarrhea and abdominal distension lasting for 9 months; in addition, the patient had symptoms of significant weight loss and anemia. The patient had visited the local clinic prior to admission and trophozoites were identified in ascites and stool. Following an anti-amebic treatment consisting of metronidazole (400 mg three times per day, orally) for 1 month by the local clinic, the symptoms were not alleviated. The patient underwent sonographic examination of the abdomen, which revealed multiple heterogeneous masses in the liver and abdomen regions, the largest measuring a maximum diameter of $12 \mathrm{~cm}$. These findings were confirmed by computed tomography (CT) of the abdomen and pelvis (Fig. 1). Laboratory results revealed that the hemoglobin count, platelet count and cancer antigen (CA) 125 concentration were $96 \mathrm{~g} / 1$ (normal, 120-160 g/l), 778x10\%/1 

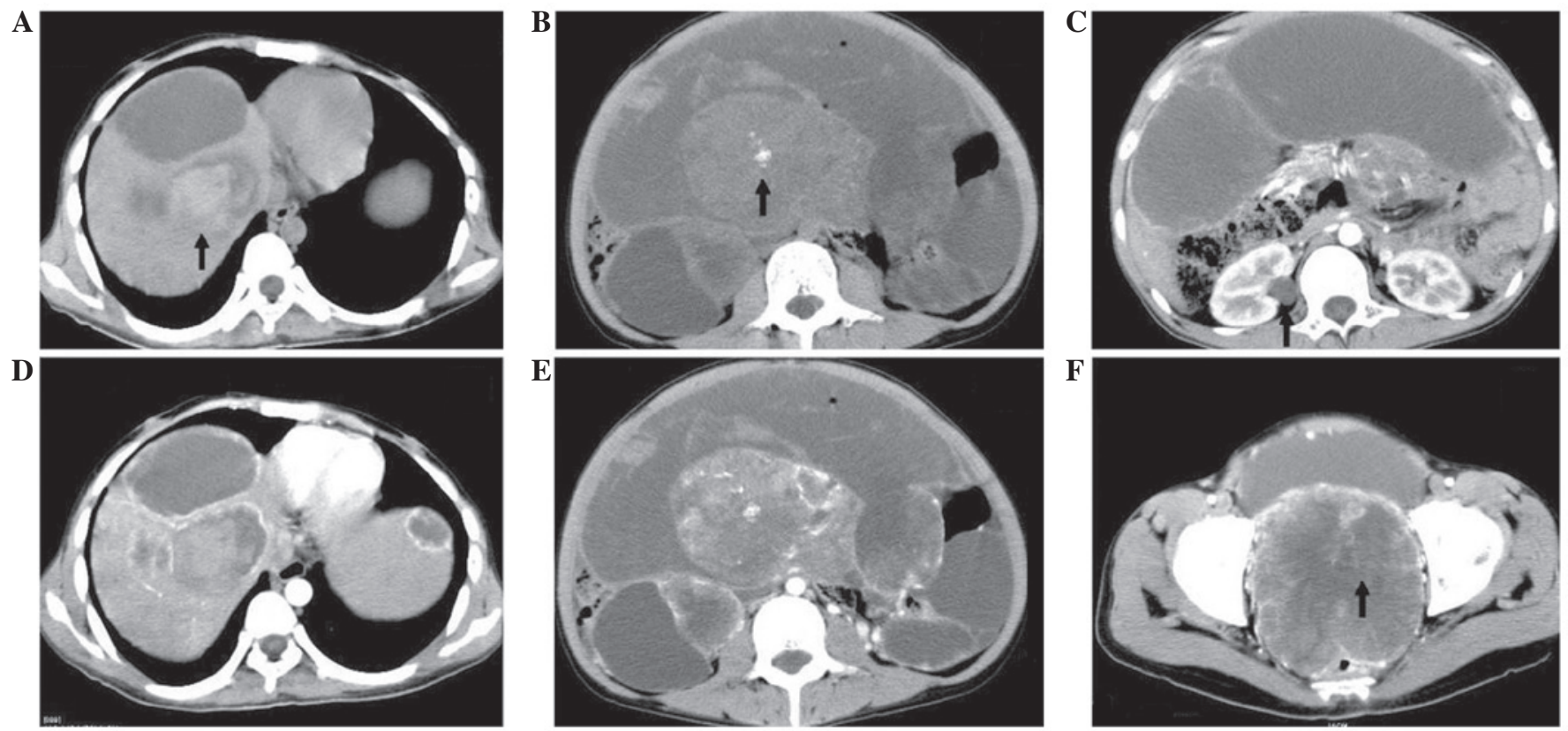

Figure 1. CT scans of the patient in Case 1. (A) A hypodense cystic-solid mixed mass was identified in the liver (arrow), with intrahepatic metastasis (B) Multiple soft tissue mass lesions with internal calcification were located in the mesentery (arrow). (C) Ascites peritoneal thickening (white arrow) and right-sided hydronephrosis (arrow) were observed. (D) Contrast-enhanced CT image [of the same view as (A)] revealed a heterogeneously-enhanced mass lesion. (E) Contrast-enhanced CT image of the abdomen [same view as (B)] revealed a heterogeneously-enhanced mass lesion. (F) Contrast-enhanced CT scan of the pelvis identified a large soft tissue mass lesion (arrow) with invasion of the bladder and colon. CT, computed topography.
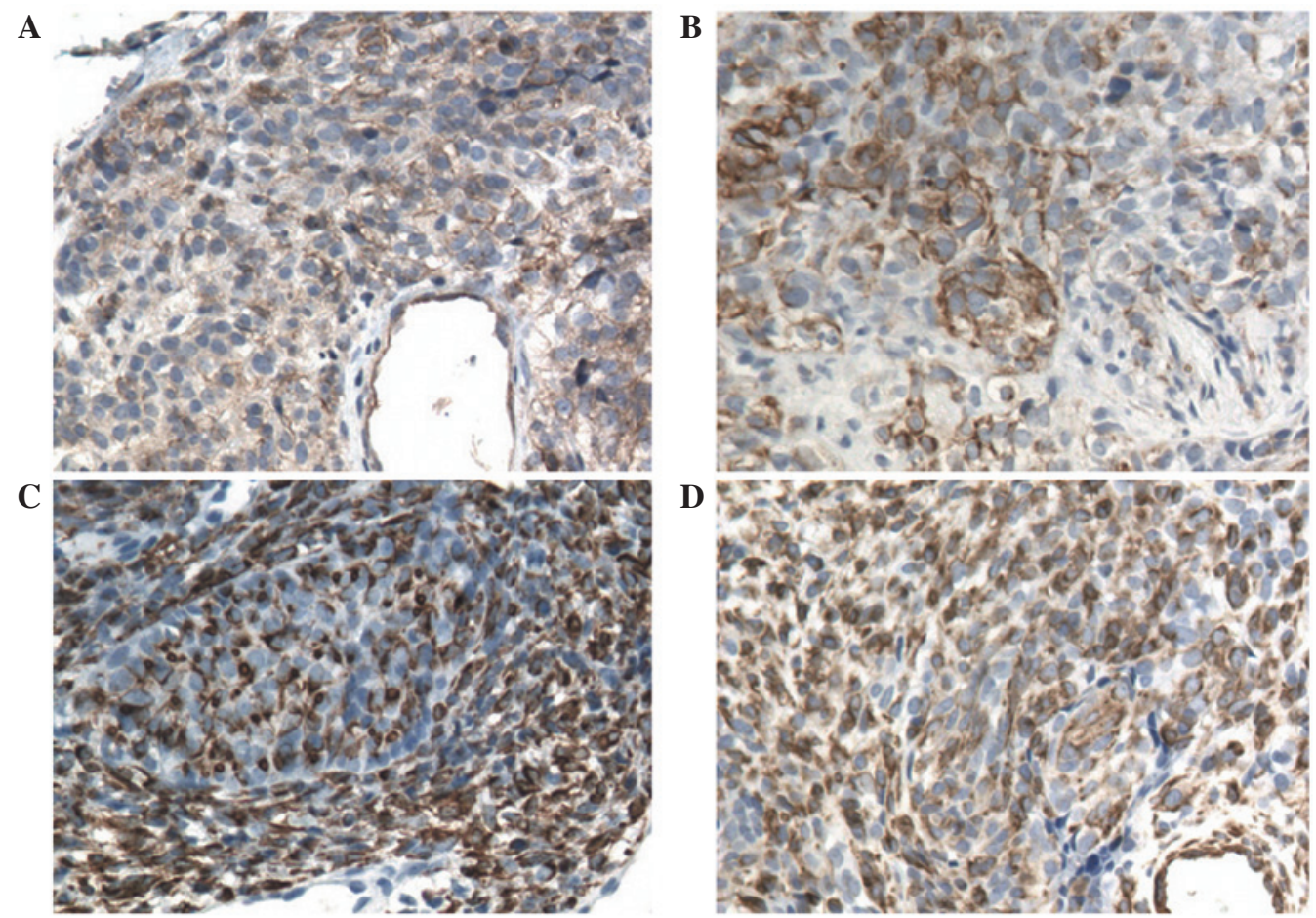

Figure 2. Immunohistochemical analysis of the biopsy specimen from the patient in Case 1. The results of the immunohistochemical analysis revealed that the membranous pattern of the biopsy specimen was positive for (A) CD99, (B) cytokeratin, (C) desmin and (D) vimentin (magnification, x400).

(normal, [100-300]x 10\%/l)and $60.9 \mathrm{U} / \mathrm{ml}$ (normal, $<25 \mathrm{U} / \mathrm{ml}$ ), respectively. Based on imaging features and laboratory data, a diagnosis of a malignant tumor with amoeba infection was made. Subsequently, an ultrasound (US)-guided percutaneous fine-needle biopsy of the abdominal mass was performed in order to histopathologically analyze the mass. The results revealed a poorly-differentiated tumor composed of clusters of small-round-cells with insufficient cytoplasm separated by a small amount of fiber. The round and spindle-shaped tumor cells exhibited a vortex-like distribution and the chromatin was evenly dispersed with inconspicuous nucleoli. The immunohistochemistry results were positive for CD99, 


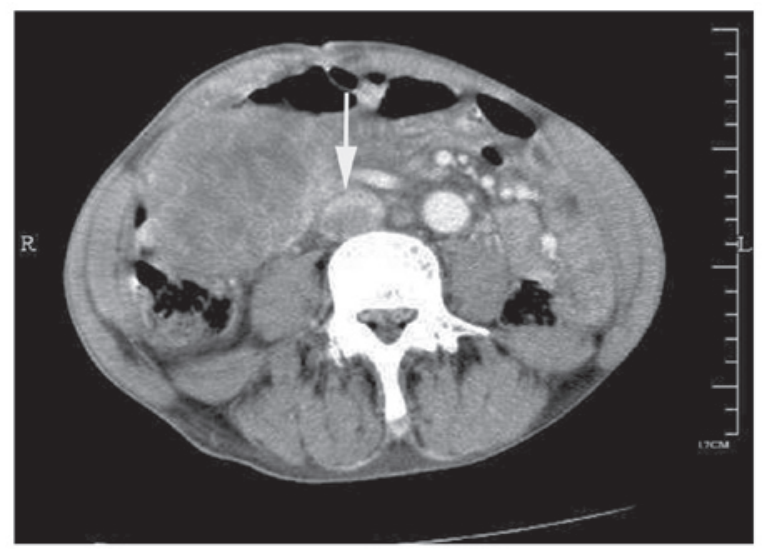

Figure 3. Case 2. Contrast-enhanced computed tomography image revealed low density in the inferior vena cava (arrow).

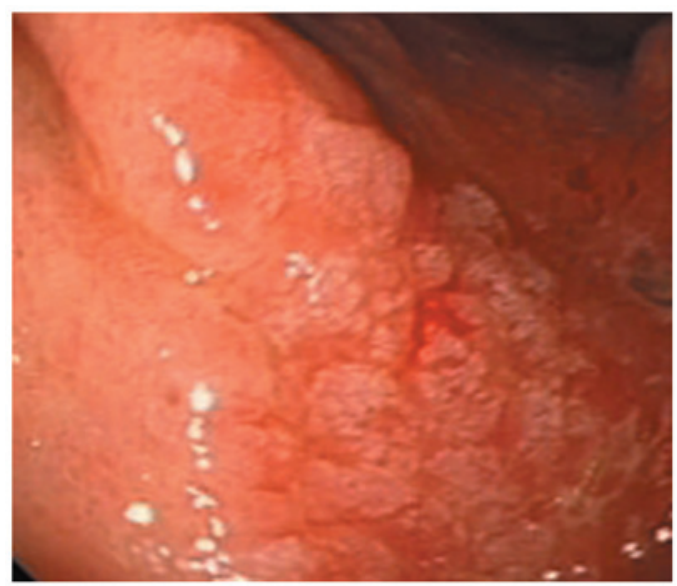

Figure 4. Case 2. Endoscopy revealed protruding lesions with erosion and ulceration on the gastric body.

cytokeratin, vimentin and desmin (Invitrogen Life Technologies, Paisley, UK; Fig. 2A-D, respectively). The Ewing Sarcoma/Wilms Tumor 1 (EWS/WT1) fusion transcript, identified by reverse transcription-polymerase chain reaction, confirmed a diagnosis of DSRCT. Chemotherapy was advised, however, the patient refused the treatment due to the financial cost of treatment and concerns regarding the potential side effects, and succumbed to his illness within 1 year of discharge from hospital.

Case 2. A 68-year-old man presented with a 20-day history of persistent abdominal pain and was admitted to the First Affiliated Hospital, College of Medicine, Zhejiang University, in February 2011. Physical examination revealed a stony hard mass in upper abdomen. Routine laboratory investigations revealed carcinoembryonic antigen and CA125 concentrations of $11.2 \mathrm{ng} / \mathrm{ml}$ (normal, 0.0-5.0 ng/ml) and $55.1 \mathrm{U} / \mathrm{ml}$, respectively. A US identified solid mass lesions in the abdominal cavity. Contrast-enhanced $\mathrm{CT}$ of the abdomen demonstrated comparable findings, as well as an inferior vena caval tumor thrombus formation (Fig. 3). An endoscopy was then performed, which revealed protruded lesions with erosion and ulcers on the gastric body (Fig. 4); in addition, the pathological results following endoscopic biopsy of the stomach indicated a diagnosis of adenocarcinoma. Subsequently, a US-guided biopsy with histological analysis of the mass provided evidence of DSRCT. The patient refused treatment due to concerns regarding side effects and financial costs, and succumbed to the disease soon after discharge from hospital.

\section{Discussion}

DSRCT is an aggressive type of malignancy, which is most commonly diagnosed in adolescents and young adults (6). At histological analysis, the tumor manifests as islands of small-round-cells encompassed by hypocellular, desmoplastic, collagenous stroma $(2,7)$. Immunohistochemical analyses of DSRCT show particular coexpression of epithelial markers (cytokeratin), mesenchymal markers (vimentin and desmin) and neural markers (neuron-specific enolase) (8). In addition, the $\mathrm{t}(11 ; 22)(\mathrm{p} 13 ; \mathrm{Q} 12)$ translocation results in the integration of the N-terminal domain of EWS to the C-terminal DNA-binding domain of WT1 resulting in the expression of an aberrant transcription factor that is involved in the pathogenesis of DSRCT (9). In the first case presented in the present study, the patient was infected with an amoeba; therefore, clinical symptoms, including diarrhea and weight loss, misled the diagnosis, resulting in delayed treatment. Due to the nonspecific manifestations of DSRCT, numerous imaging modalities are usually employed in order to evaluate patients. The primary features characteristic of DSRCT that are identified using $\mathrm{CT}$ include: Intra-abdominal soft-tissue masses located at the omental and serosal surfaces, which do not have a distinct organ of origin; solid, dominant, heterogeneous pelvic masses in the retrovesical or rectouterine regions; and concurrent metastases, which are common at the time of diagnosis, particularly those that involve the lymph nodes and liver $(10,11)$. DSRCT may be associated with ascites, hepatic metastases, adenopathies, tumor calcification and hydronephrosis due to obstructing tumor (12). In the first case described in the present study, imaging revealed several of these characteristics.

In the second case discussed in the present study, the patient was $>60$ years old, which is unusual compared with the majority of cases, which occur in young men. In addition, the imaging results of this case report revealed intra-abdominal soft-tissue masses involving the omental and serosal surfaces, which did not have a distinct organ of origin. If the biopsy of the mass was omitted from the diagnostic process, it may be concluded that the abdominal mass was homologous with gastric adenocarcinoma; however, the biopsy revealed DSRCT, which has a different prognosis and should be treated with a distinct chemotherapeutic approach.

In general, the prognosis of patients with DSRCT is poor and the ideal therapeutic modality remains to be elucidated; therefore, early diagnosis is essential. The present two cases were misdiagnosed as simple infection or gastrointestinal carcinoma due to the nonspecific clinical manifestations. Biopsy confirmed the diagnosis, however, the imaging findings were the first indication of an aggressive malignancy. Therefore, in an adolescent or young adult with multiple heterogeneous peritoneal soft tissue masses, DSRCT should be considered and biopsy is recommended. 


\section{Acknowledgements}

The present study was supported by grants from the National Science and Technology Major Project (no. 2012ZX10002004) and the Scientific Research Fund of Zhejiang Provincial Education Department (no. N20130382).

\section{References}

1. Gerald WL and Rosai J: Case 2. Desmoplastic small cell tumor with divergent differentiation. Pediatr Pathol 9: 177-183, 1989.

2. Lae ME, Roche PC, Jin L, Lloyd RV and Nascimento AG: Desmoplastic small round cell tumor: A clinicopathologic, immunohistochemical, and molecular study of 32 tumors. Am J Surg Pathol 26: 823-835, 2002.

3. Lal DR, Su WT, Wolden SL Loh KC, Modak S and La Quaglia MP Results of multimodal treatment for desmoplastic small round cell tumors. J Pediatr Surg 40: 251-255, 2005.

4. Leuschner I, Radig K and Harms D: Desmoplastic small round cell tumor. Semin Diagn Pathol 13: 204-212, 1996.

5. Mora J, Modak S, Cheung NK, Meyers P, de Alava E, Kushner B, Magnan H, Tirado OM, Laquaglia M, Ladanyi M and Rosai J: Desmoplastic small round cell tumor 20 years after its discovery. Future Oncol 11: 1071-1081, 2015.
6. Biswas G, Laskar S, Banavali SD, Gujral S, Kurkure PA, Muckaden M, Parikh PM and Nair CN: Desmoplastic small round cell tumor: extra abdominal and abdominal presentations and the results of treatment. Indian J Cancer 42: 78-84, 2005.

7. Gerald WL, Miller HK, Battifora H, Miettinen M, Silva EG and Rosai J: Intra-abdominal desmoplastic small round-cell tumor. Report of 19 cases of a distinctive type of high-grade polyphenotypic malignancy affecting young individuals. Am J Surg Pathol 15: 499-513, 1991.

8. Schröder S and Padberg BC: Desmoplsatic small-cell tumor of the peritoneum with divergent differentiation: Immunocytochemical and biochemical findings. Am J Clin Pathol 99: 353-355, 1993

9. Biegel JA, Conard K and Brooks JJ: Translocation (11;22) (p13;q12): Primary change in intra-abdominal desmoplastic small round cell tumor. Genes Chromosomes Cancer 7: 119-121, 1993.

10. Pickhardt PJ, Fisher AJ, Balfe DM, Dehner LP and Huettner PC: Desmoplastic small round cell tumor of the abdomen: radiologic histopathologic correlation. Radiology 210: 633-638, 1999.

11. Bellah R, Suzuki-Bordalo L, Brecher E, Ginsberg JP, Maris J and Pawel BR: Desmoplastic small round cell tumor in the abdomen and pelvis: Report of CT findings in 11 affected children and young adults. Am J Roentgenol 184: 1910-1914, 2005.

12. Chouli M, Viala J, Dromain C, Fizazi K, Duvillard P and Vanel D: Intra-abdominal desmoplastic small round cell tumors: CT findings and clinicopathological correlations in 13 cases. Eur J Radiol 54: 438-442, 2005. 